\title{
Enfermedades hepáticas propias del embarazo
}

\section{INTRODUCCIÓN}

La mujer gestante puede presentar cualquier tipo de enfermedad hepática (hepatitis vírica aguda, toxicidad hepática por fármacos, cálculos biliares...). Sin embargo, existen una serie de enfermedades hepáticas propias del embarazo que aparecen con la gestación y desaparecen tras el parto. La frecuencia de estas enfermedades es escasa, pero en ocasiones pueden revestir gravedad tanto para la madre como para el feto.

\section{¿QUÉ SÍNTOMAS PRODUCEN?}

Los síntomas que deben hacer sospechar estas enfermedades son el dolor localizado en el cuadrante superior derecho del abdomen o en el área gástrica, acompañado de náuseas y vómitos. Otros síntomas posibles son la ictericia (coloración amarillenta de la piel y de las mucosas) y el prurito o picor generalizado de la piel, pero ninguno de ellos es específico.

\section{¿CÓMO SE DIAGNOSTICAN?}

El diagnóstico de estas enfermedades se basa en los síntomas, el momento de la gestación en el que ocurren y en el resultado de las pruebas de laboratorio y de algunas pruebas complementarias; habitualmente una ecografía abdominal.

Las enfermedades hepáticas propias del embarazo cursan con aumento de las enzimas hepáticas: transaminasas, bilirrubina y fosfatasa alcalina (FA). En la mayoría de los casos pueden coexistir las tres alteraciones analíticas. La edad gestacional es el dato que más ayuda en el diagnóstico diferencial de estas enfermedades. En el primer trimestre el cuadro más frecuente es la hiperemesis gravídica. Entre el segundo y el tercer trimestre la colestasis intrahepática, la toxemia gravídica (preeclampsia o eclampsia) y el síndrome HELLP. En el tercer trimestre, aunque infrecuente, destaca por su gravedad la esteatosis aguda del embarazo.

\section{HIPEREMESIS GRAVIIDICA}

Habitualmente, se presenta en el primer trimestre y más raramente en el segundo. Aparece en aproximadamente en 1/100 embarazos y es más frecuente en gestantes jóvenes, primíparas y obesas. Cursa con náuseas y vómitos intensos que provocan deshidratación y desnutrición, sin otros síntomas digestivos acompañantes. En la analítica se observa un discreto incremento de los niveles de bilirrubina y de fosfatasa alcalina. El tratamiento consiste en soporte hídrico y nutricional, y sólo en casos graves se requiere alimentación mediante sonda nasogástrica o la administración de un ciclo corto de esteroides. Su pronóstico es bueno.

\section{COLESTASIS INTRAHEPÁTICA DEL EMBARAZ0 (CIE) 0 COLESTASIS GRAVIIDICA}

Generalmente se presenta en el tercer trimestre y es la segunda causa de ictericia en el embarazo, después de la hepatitis vírica aguda. Afecta a 1/2.000-8.000 embarazos y está relacionada con factores hormonales, genéticos y geográficos (muy frecuente en algunos países como Chile). Esta entidad aparece en mujeres de todas las edades, tanto primíparas como multíparas (especialmente en embarazos múltiples) y en los casos familiares puede reaparecer en gestaciones posteriores. El síntoma principal es el prurito, de predominio nocturno, que afecta sobre todo a las palmas de las manos y las plantas de los pies, aunque puede ser generalizado. La ictericia no siempre está presente y puede acompañarse de orinas oscuras (colúricas). El dolor abdominal, las náuseas y los vómitos son raros. El diagnóstico se basa en la clínica (prurito) y en las alteraciones analíiticas (incre- mento de las concentraciones de ácidos biliares en suero y aumento moderado de la bilirrubina y la FA), que desaparecen tras el parto. El pronóstico materno es bueno, pero la CIE conlleva riesgo de partos prematuros y de pérdidas fetales. El tratamiento consiste en la mejoría del prurito materno mediante diferentes fármacos (principalmente colestiramina 0 ácido ursodesoxicólico) y en la inducción del parto a partir de la semana 37. La CIE no contraindica la lactancia materna.

\section{TOXEMIA GRAVIIDICA (PREECLAMPSIA Y ECLAMPSIA)}

La preeclampsia aparece en 5-7/100 embarazos a partir de la semana 20 de gestación (entre el segundo y tercer trimestre). Se caracteriza por tensión arterial elevada (hipertensión arterial), hinchazón de las extremidades inferiores (edemas) y pérdida excesiva de proteínas en la orina (proteinuria). Cuando evoluciona a eclampsia se asocian además convulsiones y coma debido a la afectación cerebral, y en las formas graves se puede acompañar de afectación hepática. Aunque no se conoce su causa con exactitud, existen algunos factores de riesgo descritos como la hipertensión, la diabetes y las gestaciones múltiples o en edades extremas. Los síntomas y signos que indican afectación hepática son el dolor abdominal en el cuadrante superior derecho acompañado de náuseas y vómitos; y la elevación de las transaminasas (5-100 veces los valores normales). Las formas graves pueden dar lugar a complicaciones severas como el síndrome HELLP o rotura hepática. El tratamiento debe ser precoz. En las formas leves se puede intentar el reposo y fármacos para reducir la tensión arterial. En los casos graves se requiere hospitalización e interrupción del embarazo, sobre todo si la gestación ha superado la semana 34.

\section{SÍNDROME HELLP}

Aparece en aproximadamente el 4-12\% de las embarazadas con toxemia gravídica. Habitualmente se inicia entre el segundo y el tercer trimestre, pero algunos casos se pueden presentar en el postparto. Los síntomas son similares a los de la preeclampsia, a los que se unen anemia por rotura de glóbulos rojos, elevación de las enzimas hepáticas y descenso en el recuento de plaquetas. Se trata de un cuadro potencialmente grave que generalmente requiere asistencia en cuidados intensivos. Si la edad gestacional es superior a 34 semanas o aparece fallo de varios órganos, se debe interrumpir el embarazo, ya que existe riesgo fallecimiento de la madre (2-8\% de los casos). Si el cuadro no es grave y la edad gestacional es menor a 34 semanas se pueden administrar corticoides durante 48 horas para acelerar la maduración de los pulmones del feto y provocar el parto lo más cercano posible a la semana 34.

\section{ESTEATOSIS AGUDA 0 HÍGADO GRASO AGUDO DEL EMBARAZ0}

Es un proceso infrecuente del tercer trimestre del embarazo (1/7.000-16.000 embarazos), pero potencialmente grave para la madre y el feto (mortalidad materno-fetal del $20 \%$ aproximadamente). Se debe a una acumulación de grasa en el hígado y suele afectar a mujeres jóvenes, obesas y sin embarazos previos. Los síntomas iniciales consisten en náuseas/vómitos, dolor en el abdomen superior, cansancio e hipotensión; que evolucionan a alteraciones graves de la coagulación, fallo renal y de otros órganos. En la analítica suele existir anemia, descenso de las plaquetas, aumento de los glóbulos blancos y alteraciones de los parámetros de función hepática y renal. El tratamiento es similar al descrito en el síndrome HELLP y consiste en interrumpir el embarazo.

\section{S. Lorente Pérez y T. Serrano Aulló ${ }^{2}$}

${ }^{1}$ Unidad de Hepatología y Trasplante Hepático $y^{2}$ Servicio de Aparato Digestivo. Hospital Clínico Universitario Lozano Blesa. Zaragoza 